\title{
A Competência no Cotidiano: um constructo buscando uma identidade ${ }^{1}$
}

Prof. Dr. Hans-Werner Wahl ${ }^{2}$

\section{Resumo}

O presente trabalho pretende dar uma contribuição conceitual ao constructo "competência no cotidiano". Depois de uma discussão introdutória sobre as razões da crescente atenção da competência no cotidiano na Gerontologia, são esboçadas, numa primeira parte, seis tendências de pesquisa com relevância para a competência no cotidiano: 1 Pesquisa gerontológica cognitiva sobre situações no cotidiano; 2 Pesquisas sobre ADL/IADL (atividades de vida diária, instrumental de atividades de vida diária); 3 Pesquisa sobre lazer, 4 Pesquisa sobre coping; 5 Pesquisa sobre a organização temporal de atividades e 6 Pesquisa ecológico-gerontológica. Numa segunda parte, são apresentados e analisados dois modelos com forte influência na competência no cotidiano, o modelo de Margret Baltes e o modelo de Sherry Willis. Numa terceira parte é elaborada uma concepção integrada de competência no cotidiano, de modo que interrelaciona aspectos das seis tendências de pesquisa com elementos importantes dos dois modelos de competência no cotidiano.

Palavras-Chave: envelhecimento; cotidiano; competência no cotidiano.

\section{Abstract}

The goal of this paper is to contribute to conceptual issues associated with the construct of everyday competence. After the description of reasons for the $\mathbf{1} \mathbf{1}$

\footnotetext{
${ }^{1} \mathrm{O}$ artigo na língua original (alemão) foi publicado em Zeitschrift für Gerontologie und Geriatrie, Darmstad, v. 31, n. 4, p. 243-249, Aug. 1998. Agradecemos a Editora Steinkopf pela autorização da tradução e publicação. Tradução de Johannes Doll.

${ }^{2}$ Professor do Centro Alemão de Pesquisa sobre Envelhecimento na Universidade de Heidelberg.

E-mail:wahl@dzfa.uniheidelberg.de
}

Estud. interdiscip. envelhec., Porto Alegre, v.2, p.103-120, 1999. 
increased attention which everyday competence has found within gerontology, the first section of this work serves to shed light on six research traditions with relevance for everyday competence: (1) Research on everyday cognitive processes, (2) ADL/IADL research, (3) leisure time research, (4) coping research, (5) time budget research, and (6) ecological gerontology research. In the second section, two influencial models of everyday competence, namely those of Margret Baltes and Sherry Willis, are discussed. Finally, an integrative conception of everyday competence, which is aimed to combine aspects of the six research traditions with elements of the two models of everyday competence, is suggested.

Key -Words: aging, everyday life, everyday competence.

\section{Introdução e Perspectiva}

Nos últimos dez a quinze anos foram apresentados um número considerável de trabalhos conceptuais e empíricos de alto nível sobre a competência no cotidiano na velhice (dentre os quais Baltes; Mass; Wilms, 1996; Baltes; Mayr; Borchelt; Mass, 1993; Baltes; Wilms, 1995; Cornelius; Caspi, 1987; Diehl, s.d.; Marsiske; Willis, 1995; Sternberg; Wagner, 1986; Willis, 1991 e Willis, 1996). As razões do forte interesse no conceito de competência no cotidiano podem ser encontradas em diferentes níveis, seja científica, seja social, ou em relação à prática. No nível científico deve ser destacada a discrepância persistente entre resultados encontrados nos laboratórios em comparação com as capacidades no dia a dia de pessoas idosas. As pesquisas nos laboratórios demonstram, quase sem exceção, deficites relacionados com a idade ou uma diminuição das capacidades no processo de envelhecimento. Ao contrário disso encontra-se o fato da alta competência e capacidade de vivência de pessoas idosas nos seus contextos espaciais e sociais naturais (por exemplo, Kruse, 1992; Schneekloth; Ponthoff, 1993; Wahl; Wetzler (no prelo)), o que pode ser salientado, de forma especial, com o conceito de competência no cotidiano. Também recentes trabalhos voltados (de novo) para a questão do envelhecimento bom ou bem sucedido (veja, por exemplo, Baltes; Mayr; Maas; Wilms, 1993 e Baltes; Baltes, 1990) dificilmente 
conseguem referência ao envelhecimento bem sucedido, sem levar em consideração a competência no cotidiano das pessoas idosas. Especialmente o processo, descrito de Baltes; Baltes (1990), sobre a otimização seletiva com compensação como uma estratégia de um envelhecimento bem sucedido ${ }^{3}$ reflete, de forma especial, a competência no cotidiano de pessoas idosas e sua capacidade da manutenção pró-ativa de uma vida boa, apesar de todas as restrições relacionadas à velhice. A perspectiva da competência na velhice com seu destaque para as capacidades de pessoas idosas de " ( . . . ) manter uma vida autônoma, voltada para tarefas e cheia de sentido" (Kruse, 1992, p.25), formulada em resposta à perspectiva do déficit da velhice predominante por muito tempo, contribuiu também para um interesse maior dos pesquisadores para a competência no cotidiano (veja, por exemplo, Lehr; Thomae, 1991 e Olbrich, 1987). A pesquisa sobre intervenção gerontológica demonstrou as possibilidades de alterações significativas no processo de envelhecimento, por exemplo, nas áreas da autonomia básica, da capacidade cognitiva e da interação social, e provou com isso a possibilidade de modificações duradouras da competência no cotidiano (veja, por exemplo, Wahl; Tesch-Römer, 1998). Finalmente deveria o potencial interdisciplinar de pesquisa ter contribuído para a aplicação do constructo de competência no cotidiano, por exemplo, pela interrelação de variáveis médico-geriátricas, psicológicas e sociológicas nas suas capacidades explicativas relativas a competência de atuação no cotidiano de pessoas idosas (por exemplo, Baltes; Maas; Wilms; Borchlet, 1996).

Em relação as razões sociais e práticas, a mudança demográfica já conhecida leva automaticamente à questão sobre a competência no cotidiano das pessoas idosas de hoje e as de amanhã. Desta forma, a avaliação da competência no cotidiano (Que competências sobraram? Quais podem ser alcançadas de novo?) é um ponto chave na avaliação dos potenciais de uma reabilitação e para sugestões de cuidado, por exemplo, em relação a futura situação de moradia depois de uma situação de crise por causa da saúde. Da

\footnotetext{
${ }^{3}$ NT: veja a respeito deste modelo em língua portuguesa: Staudinger, U. M.; Marsiske, M; Baltels, P. B. Resiliência e Níveis de Capacidade de Reserva na Velhice: perspectivas da teoria de curso de vida. In: Neri, A. L. (Org.) Psicologia do Envelhecimento. Campinas, Papirus, 1995. p.195-228.
} 
mesma maneira, é relevante que se trate hoje, e ainda mais amanhã, de "idosos novos" que possuem novas competências no cotidiano, por exemplo, em relação ao uso de um carro próprio e, com isso, uma maior mobilidade, ou nas formas de lidar com novas técnicas (Wahl; Saup, 1994).

O objetivo deste trabalho teórico é, a partir deste pano de fundo, analisar o conceito de competência no cotidiano na sua aplicabilidade a pessoas idosas. $\mathrm{Na}$ primeira parte do mesmo, são esboçadas tendências de pesquisa na Gerontologia com relevância direta para a competência no cotidiano. Na segunda parte, são discutidas duas concepções centrais e influentes de competência no cotidiano, uma de Willis (1991), outra de M. Baltes et al. (1996). Na terceira parte, finalmente, é proposta uma concepção integrante do conceito "competência no cotidiano" interrelacionando elementos essenciais das tendências de pesquisa bem como aspectos centrais dos dois modelos.

\section{Sobre a Identidade do Constructo Competência no Cotidiano I:} tendências de pesquisa em Gerontologia com relevância direta

São seis as tendências de pesquisa em Gerontologia que, de forma direta, alimentam através das suas concepções e resultados o contructo competência no cotidiano: 1 Pesquisa gerontológica cognitiva sobre situações no cotidiano; 2 Pesquisa sobre ADL/IADL (activities of daily life, instrumental activities of daily life $e^{4}$ ); 3 Pesquisa sobre lazer; 4 Pesquisa sobre coping; 5 Pesquisa sobre a organização temporal de atividades e 6 Pesquisa ecológico-gerontológica. Estas tendências são esboçadas a seguir.

\subsection{Pesquisa Gerontológica Cognitiva}

É vista, por um lado, como uma das mais avançadas tendências de pesquisa da Gerontologia psicológica em relação à metodologia e aos resultados. Por outro lado, levantou-se nos últimos anos uma certa crítica à pesquisa gerontológica cognitiva tradicional, alertando que sabemos relativamente pouco sobre os processos e competências de pessoas idosas para resolver os problemas do cotidiano (por exemplo, Park, 1992; Wahl, 1990a; Wahl, 1990b e Willis, 1996). Em seguinda foi apresentado um considerável número de trabalhos

\footnotetext{
${ }^{4}$ N. T. Atividades de via diária, atividades instrumentais de vida diária.
} 
voltados especificamente para os processos de solução de problemas do cotidiano, baseados em instrumentos bem mais próximos do dia a dia e com forte relação aos desafios da atuação no cotidiano (por exemplo, Cornelius; Caspi, 1987; Diehl; Willis; Schaier, 1995; Marsiske; Willis, 1995; Sternberg; Wagner, 1986).

\subsection{ADL, IADL}

Existe uma longa tradição de trabalhos que se referem às assim chamadas atividades de vida diária (ADL, IADL). De forma particular na literatura das ciências sociais, da epidemiologia e da geriatria, as atividades de vida diária se transformaram em uma das mais importantes variáveis para caracterizar a qualidade de vida na velhice:

a) como indicador de sucesso de uma reabilitação ou de necessidade de cuidado;

b) como variável da saúde funcional e sua alteração em relação à coorte e,

c) como elemento central da assim chamada expectativa de vida ativa (active life expectancy) (por exemplo, Crimmins; Hayward; Saito,1996; Wahl 1988; Wolinsky; Fitzgerald; Johnson, 1993). Do ponto de vista atual, dificilmente pode ser superestimado o valor desta tendência de ADL/IADL em relação a uma mudança da "visão diagnóstica" da velhice para uma perspectiva funcional e orientada para as competências e déficitis concretos no cotidiano das pessoas idosas.

\subsection{Atividades de Lazer}

Observam-se atividades intensivas de pesquisa sobre as atividades de lazer na idade avançada que têm também muito a ver com as competências no cotidiano de pessoas idosas (por exemplo, Tokarski, 1989). Um aspecto central no tempo disponível e livre do cotidiano de pessoas idosas parece ser, entre outras, a realização de hobbys, atividades culturais e esportivas. O específico desta tendência de pesquisa é o destaque dos elementos de desenvolvimento e crescimento e dos estímulos da competência no cotidiano na velhice e não exclusivamente os aspectos funcionais para manter a independência na condução da vida, como sugere a perspectiva da ADL/IADL. 


\subsection{Pesquisa sobre coping}

Sem dúvida, também os trabalhos de Hans Thomae direcionaram os olhares para o cotidiano das pessoas idosas. Thomae (1996 e 1998; veja também Lehr; Thomae, 1991) elabora o significado do objeto de análise, "cotidiano", de forma explícita, com o conceito de "técnicas do ser" (Daseinstechniken) (Thomae, 1968), mais tarde com as "formas de reação" (Reaktionsformen) (Thomae, 1996), ele coloca as formas cotidianas de enfrentar os desafios da vida e do envelhecimento no centro do seu trabalho. Mas também a pesquisa sobre coping em geral não se orienta exclusivamente pelos "grandes" acontecimentos críticos de vida, mas também pelos pequenos problemas do dia a dia (daily hassles) (por exemplo, Tesch-Römer; Salewski; Schwarz 1997).

\subsection{Organização Temporal das Atividades}

Já há um bom tempo que são apresentados trabalhos a respeito da assim chamada pesquisa sobre a organização temporal de atividades e que, desde os anos 70, cada vez mais podem ser observados também na Gerontologia. Devese lembrar o trabalho de Moss; Lawton (1982) baseado numa metodologia yesterday; o trabalho de M. Baltes; Wahl; Schmid-Furstoss (1990) com base na análise quantitativa de diários e, ultimamente, os trabalhos no contexto da pesquisa de Berlin sobre envelhecimento (Baltes; Mayr; Borchelt; Maas; Wilms, 1993). Esta tendência de pesquisa se caracteriza pelo fato de encarar o cotidiano inteiro na velhice e pela descrição diferenciada a partir do tripé clássico desta tendência (Que atividade quando? Em que contexto social? Em que lugar?). Competências separadas do cotidiano se juntam a uma imagem geral, a qual reflete a dinâmica do stream of behavior cotidiano.

\subsection{Pesquisa Ecológica-gerontológica}

Finalmente, devem ser nomeadas as variadas contribuições para uma Gerontologia ecológica, um campo de pesquisa que coloca no centro da sua atenção especialmente a investigação do mundo do dia a dia no qual a pessoa idosa sempre está interligada, de uma forma indissolúvel, com as condições contextuais sociais, espaciais e culturais (Saup, 1993; Wahl, 1992; Wahl; Saup, 1994). Assim, esta tendência de pesquisa pode fornecer a necessária perspectiva contextual da competência no cotidiano. Por exemplo, é necessário observar a importância significante do ambiente espacial, principalmente a moradia e o 
contexto da moradia, como fator de influência à competência no cotidiano (por exemplo, Braderburg, 1996; Wahl, 1997; Wahl; Wetzler (no prelo)). Da mesma forma, padrões de interação podem fortalecer ou enfraquecer a competência no cotidiano, como por exemplo através de um cuidado exagerado (Wahl, 1991).

Na Tabela são resumidas as contribuições das seis tendências de pesquisa para o constructo atividade de vida diária. Com isso, porém, a identidade do constructo é esboçada somente de forma muito geral. Em um próximo passo pretendemos aproveitar de dois modelos da competência no cotidiano na velhice para esclarecer sua identidade ainda mais.

\section{Tabela -Tendências de Pesquisa da Gerontologia e sua Importância} para a Competência no Cotidiano na Velhice

\begin{tabular}{l|l}
\hline $\begin{array}{l}\text { Tendências de Pesquisa da } \\
\text { Gerontologia }\end{array}$ & $\begin{array}{l}\text { Importância para a Competência no } \\
\text { Cotidiano na Velhice }\end{array}$ \\
\hline $\begin{array}{l}\text { Pesquisa gerontológica cognitiva } \\
\text { sobre situações no cotidiano }\end{array}$ & $\begin{array}{l}\text { Capacidade cognitiva como fundamento da } \\
\text { competência no cotidiano }\end{array}$ \\
\hline Pesquisa sobre ADL/IADL & $\begin{array}{l}\text { Atividades fundamentais do dia a dia como } \\
\text { aspectos importantes da competência no } \\
\text { cotidiano }\end{array}$ \\
\hline Pesquisa sobre lazer & $\begin{array}{l}\text { Competência no cotidiano como realização } \\
\text { do tempo "livre" e como manutenção de } \\
\text { contatos sociais e interesses }\end{array}$ \\
\hline Pesquisa sobre coping & $\begin{array}{l}\text { Competência no cotidiano como } \\
\text { enfrentamento e superação de desafios do } \\
\text { dia a dia }\end{array}$ \\
\hline $\begin{array}{l}\text { Pesquisa sobre a organização } \\
\text { temporal de atividades }\end{array}$ & $\begin{array}{l}\text { Interrelação de vários aspectos da } \\
\text { competência no cotidiano no "Stream of } \\
\text { Behavior" do dia a dia }\end{array}$ \\
\hline Pesquisa ecológico-gerontológica & $\begin{array}{l}\text { Destaque da contextualidade da } \\
\text { competência no cotidiano e uma } \\
\text { preocupação metódica com o cotidiano }\end{array}$ \\
\hline
\end{tabular}

Estud. interdiscip. envelhec., Porto Alegre, v.2, p.103-120, 1999. 
3 Sobre a Identidade do Construto Competência no Cotidiano II: comparando modelos teóricos.

\subsection{O Modelo de Willis}

Willis (Willis, 1991) pretende com seu modelo superar a pobreza teórica da literatura sobre ADL/IADL, notada apesar de todos os méritos (veja anteriormente). Competência no cotidiano fica definida da seguinte forma: "As atividades de vida diária significam habilidades ou o potencial de pessoas adultas de realizar de forma adequada aquelas atividades consideradas essenciais para uma vida autônoma." (p. 81). De forma especial, Willis se refere aos sete aspectos de IADL: lidar com questões financeiras, tomar remédios, telefonar, fazer compras, cozinhar, arrumar a casa e caminhar uma certa distância fora de casa.

A assim definida competência no cotidiano é composta por condições antecedentes, componentes, mecanismos e efeitos (veja Fig. 1). Enquanto as condições antecedentes, sejam elas individuais (por exemplo, capacidade cognitiva) ou sócio-culturais (por exemplo, estereotipos da velhice) são vistas

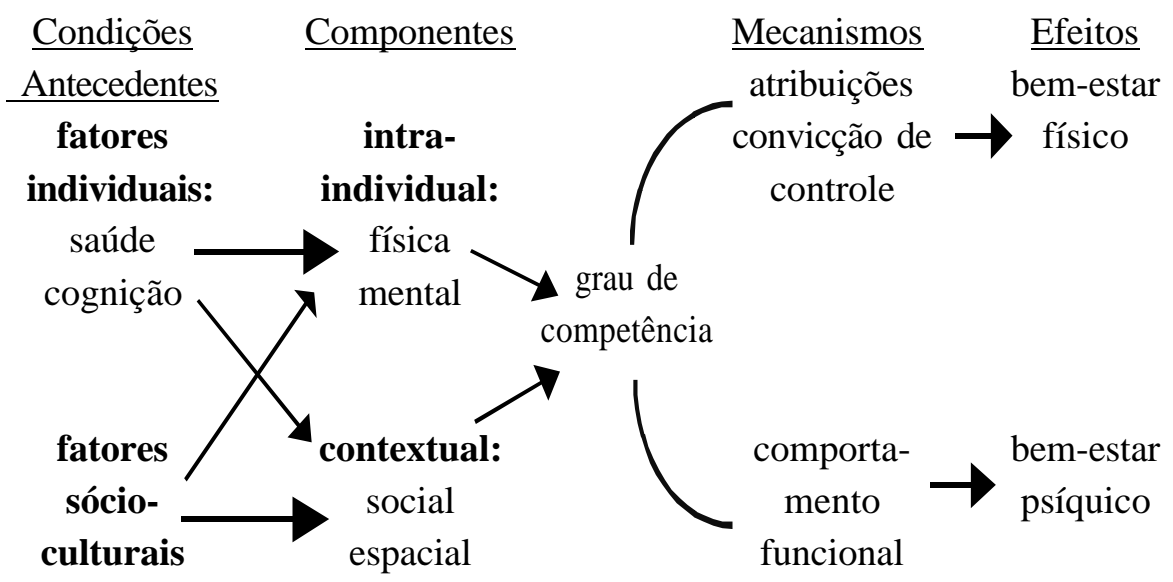

Figura 1 - O Modelo de Competência no Cotidiano de Willis (apud Willis 1991, p. 82) 
como fatores influentes de forma transcontextual, pressupõe-se que existem certos componentes intraindividuais e contextuais, portanto constelações concretas entre pessoa e ambiente, que produzem efeitos em relação a certas facetas ou aspectos específicos da competência no cotidiano. Além do mais se espera que às condições antecendentes e aos componentes se juntem mecanismos específicos e somente desta forma consegue-se uma determinada competência no cotidiano ("level of competence"). Segundo Willis, tais mecanismos são, por exemplo, a convicção de controlar sua vida ou atribuições, mas também a realização regular de determinadas atividades no sentido de um treinamento constante. Finalmente pode-se prever efeitos da competência no cotidiano em relação ao bem-estar psíquico e físico.

\subsection{O Modelo de M. Baltes et al.}

A competência no cotidiano deste modelo é definida como a realização efetiva de atividades cotidianas e a superação dos desafios do dia a dia. Porém, estes desafios não ficam restritos a algumas atividades do dia a dia no sentido das listas de ADL/IADL. São levados em conta em relação à competência no cotidiano capacidades básicas como o auto cuidado e a alimentação regular, aptidões instrumentais do cotidiano bem como o comportamento social e o comportamento em relação ao lazer.

Além disso, se diferencia entre três perspectivas a respeito da competência no cotidiano (veja Fig. 2): primeiro uma perspectiva de capacidade, na qual é vista somente a realização ou não-realização (objetivamente) de certas atividades; segundo uma perspectiva de maestria $a^{5}$ que considera a percepção subjetiva de conseguir lidar com as atividades do dia a dia, aspectos da competência no cotidiano; terceiro, uma perspectiva de adequação onde é observada a adequação entre a competência no cotidiano e as condições e os desafios do contexto. Um enfoque especial do trabalho de Baltes et al. consiste na elaboração das dimensões empíricas da competência no cotidiano, o que leva a uma diferenciação entre uma competência básica (por exemplo, conseguir alimentar-se, lavar-se, vestir-se) e uma competência ampliada.

\footnotetext{
5 N.T. O autor chama de perspectiva "mastery"

${ }^{6}$ N.T. "adaptive fit"

Estud. interdiscip. envelhec., Porto Alegre, v.2, p.103-120, 1999.
} 


\section{perspectiva de capacidade \\ engajamento em determinadas \\ atividades}

\begin{tabular}{|c|}
\hline perspectiva de maestria \\
percepção subjetiva de \\
conseguir lidar com suas \\
capacidades \\
\hline
\end{tabular}

perspectiva de adequação ${ }^{6}$

adequação entre capacidade $\mathrm{e}$ desafio contextual competência básica no

cotidiano

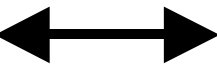

competência ampliada

no cotidiano

Figura 2 - O Modelo de Competência no Cotidiano de Baltes et al. (apud Baltes, Cartensen 1996)

\subsection{Comparação dos modelos}

Em relação à definição dos limites (inclusão e exclusão de aspectos) da competência no cotidiano, Willis (1991) favorece uma compreensão relativamente estreita de competência no cotidiano, bem no sentido da linha tradicional de ADL. Margaret Baltes e colegas opõem a isso uma perspectiva mais ampla que inclui as atividades de lazer e de relações sociais. Levando em conta as tendências de pesquisa descritas com relevância para a competência no cotidiano, parece-nos a compreensão mais ampla a mais convincente, porém ainda não abrangente o suficiente. Assim, pode-se questionar de que forma são considerados o lado cognitivo da realização das tarefas do cotidiano, os processos de solução de problemas diários e a superação de desafios cognitivos como parte da competência no cotidiano (veja Willis, 1996). Da mesma forma, pode se perguntar a posição dos dois modelos em relação aos processos de coping e referente aos múltiplos esforços compensatórios de pessoas idosas, tematizados ultimamente em dois trabalhos de bastante repercussão (Bäckman; Dixon, 1992; Dixon; Bäckman, 1995). Segundo nossa opinião, estes processos

\footnotetext{
${ }^{6}$ NT. "adaptive fit"
} 
devem ser considerados também como aspectos da competência no cotidiano. Pelo menos parcialmente fica aberto, nos dois modelos, a questão de que forma aspectos de desenvolvimento da competência no cotidiano são considerados, quer dizer, uma perspectiva que entende competência no cotidiano não somente na sua funcionalidade no dia a dia da velhice, mas também como estímulo para o comportamento e a vivência e, desta maneira, como dinâmica básica de um "bom envelhecimento" (veja White, 1959). Através da inclusão de atividades de lazer e atividades sociais, o modelo de Baltes considera estes fatores mais do que o modelo de Willis.

Nos dois modelos o papel do contexto sócio-cultural para formação de competência no cotidiano (veja também Willis et al., 1997) é destacado. Também em ambos os modelos percebe-se a necessidade de incluir tanto parâmetros objetivos, quanto a avaliação subjetiva da competência no cotidiano. De forma especial, estes modelos destacam a importância de uma perspectiva contextual da competência no cotidiano: Willis faz isso através do "componente contextual”, Baltes através da perspectiva de adaptação.

No modelo de Willis, a competência no cotidiano é destacada como condição importante para o bem-estar físico e psíquico. Willis recorre para tal a resultados da literatura sobre ADL demonstrando, que a competência, especialmente a competência nos IADL são os melhores preditores para a institucionalização e mortalidade. Em comparação com isso, a competência no cotidiano representa no modelo de Baltes mais uma variável dependente, diferenciada em competência básica e ampliada. Por outro lado, no modelo de Baltes a competência no cotidiano pode ser vista como preditor e como acontecimento processual em relação a resultados de processos de envelhecimento. Por exemplo, um desenvolvimento demencial pode se anunciar já desde cedo através de dificuldades específicas na competência básica, um desenvolvimento depressivo pode ser percebido por alterações na competência ampliada.

4 Sobre a Identidade do Constructo Competência no Cotidiano III: Tentativa de uma Concepção Integrada

$\mathrm{Na}$ parte anterior, foram agrupadas tendências de pesquisa com 
relevância direta para o constructo de competência no cotidiano e juntamos estas tendências através de dois modelos. Propomos agora, aproveitando de outros pensamentos na literatura específica (Diehl (no prelo), a seguinte concepção integrante.

A competência no cotidiano (de pessoas idosas) abrange aptidões físicas, psíquicas (cognitivo-emocional) e sociais, as quais, em interação entre si e em interação com condições e barreiras do ambiente, levam a uma vivência concreta e a um comportamento em contextos cotidianos relevantes, segundo expectativas e objetivos próprios e da sociedade.

O objetivo desta concepção é manter os elementos chaves dos dois modelos de Baltes e Willis e adicionar aspectos das tendências de pesquisa relevantes para a competência no cotidiano. Segundo esta concepção, a competência no cotidiano se baseia, como destacado por Baltes e Willis, em aspectos físicos, psíquicos e sociais da pessoa, os quais devem ser vistos sempre em interrelação com as possibilidades e condições do ambiente. Desta forma, os aspectos psíquicos incluem explicitamente também capacidades cognitivas no sentido mais estreito como processos de solução de problemas voltados para o dia-a-dia, bem como processos cognitivo-emocionais de vencer desafios no sentido das pesquisas sobre coping (Tesch-Römer; Salewski; Schwarz, 1997) ou orientados nas formas de reação de Thomae (1996).

O ambiente, tanto no sentido social, quanto espacial, é compreendido como um fator de influência que facilita ou dificulta a competência no cotidiano. Nisso foi referido aos resultados da Gerontologia ecológica que sublinha que especialmente pessoas idosas podem se tornar, muitas vezes, "vítimas" de condições adversas do ambiente (veja a hipótese de docility $^{7}$ ), mas que, por

\footnotetext{
${ }^{1}$ NT. A hipótese de "docility” é uma das contribuições centrais da gerontologia ecológica. Ela supõe que as pessoas procuram um certo equilíbrio entre as exigências do ambiente e os recursos próprios. Isso significa que o ambiente (environment) exerce uma certa pressão para que as pessoas se adaptem a ela (por exemplo, às condições de moradia) ou provoquem ativamente mudanças no ambiente (hipótese de "environmentalproactivity", por exemplo: mudam a moradia ou procuram uma outra mais adequada). A hipótese de "environmental-docility" vai dizer que mesmo a pessoa dispondo de recursos as condições do ambiente é que são determinantes. (veja, Olbrich, E. Menschengerechte Umweltgestaltung. Schnittstelle zwischen Person und Umgebung. Zeitschrift für Gerontologie und Geriatrie, v.29, p.257-266, 1996.) Recursos aqui não significam somente recursos financeiros, mas muito mais recursos pessoais no sentido de capacidade de atuação, recursos cognitivos, de personalidade, etc.
} 
outro lado, elas podem ter muita criatividade no uso de possibilidades e condições do ambiente no sentido de realizar objetivos próprios; com isso, elas demonstram um alto grau de "pro-atividade" (Lawton, 1985; Olbrich, 1987; Parmelee; Lawton, 1990).

Competência no cotidiano se manifesta na vivência e no comportamento em contextos no dia a dia, no qual tanto o comportamento projetado, quanto a relevância do contexto se orientam não só segundo os desejos das pessoas idosas, mas também segundo os objetivos e as expectativas da sociedade. Com isso pretende-se salientar que a competência no cotidiano deve ser entendida sempre como o resultado de uma interrelação complexa de fatores objetivos da pessoa (por exemplo, saúde), com objetivos e expectativas próprias, mas também de condições pré-existentes da sociedade. Autonomia nas ADLs e IADLs é um exemplo típico para uma tal interrelação.

A formulação aberta de "vivência e comportamento" deixa bastante espaço para as múltiplas facetas do cotidiano, abrangendo tanto ADLs e IADLs, quanto atividades de lazer, experiências sociais ou a realização de desafios cotidianos, como, por exemplo, o planejamento e a realização de uma necessária mudança de moradia. A formulação inclui tanto o comportamento, quanto a sua qualidade cognitivo-emocional, o que acentua os aspectos de estimulação e de desenvolvimento da competência no cotidiano.

Finalmente, a concepção proposta deixa conscientemente aberta o status teórico da competência no cotidiano no sentido de uma variável independente, moderadora ou dependente. Supõe-se que a competência no cotidiano pode assumir cada uma destas três variantes no "stream of behavior" da vivência e atuação cotidiana. $\mathrm{O}$ interesse investigativo determina a perspectiva analítica e não o constructo em si.

\section{Considerações Finais}

A linha de pensamento apresentada neste trabalho deve ter deixado claro a minha opinião: a competência no cotidiano não é um "simples" constructo que pode ser operacionalizado de uma única forma "correta". Muito pelo contrário, trata-se de um constructo "perspectívico", que abre em primeiro lugar um olhar sobre o cotidiano de pessoas idosas. $\mathrm{O}$ foco dentro deste olhar 
pode ser bem diferente, e não existe nenhuma primazia em relação a uma determinada disciplina ou subdisciplina científica. Por outro lado, o conceito de competência no cotidiano exerce uma certa atração para diferentes tendências de pesquisa as quais, até agora, existiram relativamente desconectadas uma da outra. Isso vale mesmo para uma única disciplina como, por exemplo, a Psicologia. Desta forma, e nossa concepção de trabalho aqui deveria fortalecer isso, o constructo de competência no cotidiano abre caminhos, para interrelacionar, desde o início, diferentes tendências de pesquisa empírica, como a análise de solução de problemas no cotidiano (pesquisa gerontológica cognitiva), o enfrentamento de desafios emocionais diárias (coping) e a realização concreta no "stream of behavior" diário (pesquisa de lazer). A referida interrelação deve ser entendida somente como exemplo, pois outras formas e relações, em diferentes direções, podem ser pensadas. Poder-se-ia, por exemplo, investigar a questão: se as estratégias cognitivas de pessoas idosas para a solução de situações do cotidiano estão em alguma relação com as formas de enfrentar desafios emocionais (perspectiva das pesquisas sobre coping) e como a interação das duas influencia as atividades diárias no tempo livre. Questões deste tipo possuem, segundo minha perspectiva, um carater novo e foram, até agora, pouco trabalhadas. Uma tal junção de linhas de pesquisa, já dentro de uma disciplina (no caso, a Psicologia) e ainda muito mais numa perspectiva interdisciplinar poderia ser um avanço da Gerontologia levando como referência o dia a dia e a competência no cotidiano das pessoas idosas. Com isso seria possível colocar um contraponto a uma especialização cada vez maior das pesquisas em Gerontologia.

\section{Referências Bibliográficas}

1 BALTES, M. M.; CARSTENSEN, L. L. Gutes Leben im Alter: Überlegungen $\mathrm{zu}$ einem prozeßorientierten Metamodell erfolgreichen Alterns. Psychologische Rundschau, Goettingen, v.47, p.199-215, 1996.

2 BLATES, M. M.; MAAS, I.; WILMS, H-U.; BORCHLET, M. Alltagskompetenz im Alter: Theoretische Überlegungen und empirische Befunde. In: MAYER, K.U.; BALTES, P.B. (Hrsg). Die Berliner Altersstudie. Berlim, Akademie Verlag, 1996, p.525-542. 
3 BALTES, M. M.; MAYR, U.; BORCHELT, M.; MAAS, I.; WILMS, U. Everyday Competence in Old and Very Old Age: an inter-disciplinary perspective. Ageing and Society, Cambridge, v.13, p.657-680, 1993.

4 BALTES, M. M.; WAHL, H-W.; SCHMID-FURTOSS, U. The Daily Life of Elderly Germans: activity patterns, personal control, and functional health. Journal of Gerontology: Psychological Sciences, Washington, D. C., v.4, p.173-179, 1990.

5 BALTES, M. M.; WILMS, H. U. Alltagskompetenz im Alter. In: OERTER, R.; MONTADA, L. (Hrsg.). Entwicklungpsychologie. 3. Aufl. Weinheim, Psychologie Verlags Union, 1995. p.1127-1136

6 BALTES, P. B.; BALTES M. M. Psychological Perspectives on Successful Aging: the model of selective optimization with compensation. In: BALTES, P. B.; BALTES M. M. (Eds.). Successful Aging. Perspectives from the Behavioral Sciences. Cambridge, University Press, 1990, p. $1-34$

7 BÄCKMAN, L.; DIXON, R. A. Psychological Compensation: a theoretical framework. Psychological Bulletin, Washingtom, D. C., v. 112, p.259283, 1992.

8 BRANDERBURG, H. Formen der Lebensführung im Alltag bei hilfeund pflegebedürftigen älteren Menschen. Roderer, Regensburg., 1996.

9 CORNELIUS, S.; CASPI, A Everyday Problem Solving in Adulthood and Old Age. Psychology and Aging, Washington, D. C., v.2, p.144-153, 1987.

10 CRIMMINS, E. M.; HAYWARD, M. D.; SAITO, Y. Differentials in Active Life Expectancy in the Older Population of the United States. Journal of Gerontology: Social Sciences, Washington, D. C., v. 51B, p.111120, 1996.

11 DIEHL, M. Everyday Competence in Later Life: current status and future directions. The Gerontologist, Washington, D.C. [no prelo]

12 DIEHL, M.; WILLIS, S.; SCHAIE, K. W. Everyday Problem Solving in Older Adults: observational assessment and cognitive correlates. Psychology and Aging, Washington, D. C., v.10, p.478-491, 1995. 
13 DIXON, R. A.; BÄCKMAN, L. Compensating for Psychological Deficits and Declines. Managing Losses and Promoting Gains. Mahwah, NJ, Erlbaum, 1995.

14 KRUSE, A. Kompetenz im Alter in ihren Bezügen zur objektiven und subjektiven Lebenssituation - Darstellung eigener Ergebnisse der Untersuchung. In: Schütz R. M., Kuhlmey, A., Tews, H. P. (Eds.) Altern in Deutschland. Berlin, Theuberger Verlag, 1992. p. 25-32.

15 LAWTON, M. P. The Elderly in Context. Environment and Behavior, Beverly Hills, Cal., v.17, p.501-519, 1985.

16 LEHR, U.; THOMAE, H. Alltagspsychologie. Aufgaben, Methoden, Ergebnisse. Darmstadt, Wissenschaftliche Buchgesellschaft, 1991.

17 MARSISKE, M.; WILLIS, S.L. Dimensionality of everyday Problem Solving in Older Adults. Psychology and Aging, Washingotn, D. C., v.10, p.269-283, 1995.

18 MOSS, M.; LAWTON, M. P. Time Budgets of Older People: a window on four life styles. Journal of Gerontology, Washington, D. C., v.37, p.576-582, 1982.

19 OLBRICH, E. Kompetenz im Alter. Zeitschrift für Gerontologie, Darmstadt, v.20, p.319-330, 1987.

20 PARK, D.C. Applied Cognitive Aging Research. In: Craik FIM, Salthouse TA (Eds.). The Handbook of Aging and Cognition. Hillsdale, NJ, Erlbaum, 1992. p. 449-463.

21 PARMELEE, P. A.; LAWTON, M. P. The Design of Special Environment for the Aged. In: Birren JE, Schaie KW (Eds.). Handbook of the Psychology of Aging. 3. ed. New York, Academic Press, 1990. p. 465489.

22 SAlthouse, T. A. Cognitive Competence and Expertise in Aging. In Birren JE, Schaie K W (Eds) Handbook of the Psychology of Aging. 3.ed. San Diego, Academic Press, 1990. p.310-319.

23 SAUP, W. Alter und Umwelt. Eine Einführung in die Ökologische Gerontologie.Stuttgart, Kohlhammer, 1993. 
24 SCHNEEKLOUTH, U.; POTTHOFF, P. Hilfe- und Pflegebedürftige in privaten Haushalten. Bericht zur Repräsentativerhebung im Forschungsprojekt "Möglichkeiten und Grenzen selbständiger Lebensführung". In: AUFTRAG des Bundesministeriums für Familie. Stuttgart, Kohlhammer, 1993.

25 STERNBERG, R.; WAGNER, R. K. (Eds.). Practical Intelligence - nature and origin of competence in the everyday world. New York, Cambridge University Press, 1986.

26 TESCH-RÖMER, C.; SALEWSKI, C.; SCHWARZ, G. (Hrsg.). Psychologie der Bewältigung. Weinheim, Psychologie Verlags Union, 1997.

27 THOMAE, H. Das Individuum und seine Welt. Göttingen, Hogrefe, 1968.

28 THOMAE, H. Das Individuum und seine Welt. 3. Aulf. Göttingen, Hogrefe, 1996.

29 TOKARSKI, W. Freizeit- und Lebensstile älterer Menschen. Kasseler Gerontologische Schriften. Kassel, 1989.

30 WAHL, H-W.(Alltägliche Aktivitäten bei alten Menschen: Konzeptuelle und methodische Überlegungen. Zeitschrift für Gerontopsychologie und -psychiatrie, Bern, v.1, p.75-81, 1988.

31 WAHL, H-W. Auf dem Wege zu einer alltagsbezogenen Gerontopsychologie. Teil I: Konzeptuelle und methodologische Rahmenbedingungen. Zeitschrift für Gerontopsychologie und -psychiatrie, Bern, v.3, p.1323, 1990a.

32 WAHL, H-W. Auf dem Wege zu einer alltagsbezogenen Gerontopsychologie. Teil II: Beispielhafte Ansätze aus den Bereichen Kompetenz, Coping, Kontrolle und Attribution. Zeitschrift für Gerontopsychologie und -psychiatrie, Bern, v. 3, p.191-200, 1990 b.

33 WAHL, H-W. "Das kann ich allein!” Selbständigkeit In: ALTER: Chancen und Grenzen. Huber, Bern, 1991.

34 WAHL, H-W. Ökologische Perspektiven in der Gerontopsychologie: Ein Blick in die vergangenen drei Jahrzehnte und in die Zukunft. Psychologische Rundschau, Goettingen, v.43, p.232-248, 1992. 
35 WAHL, H-W. Ältere Menschen mit Sehbeeinträchtigung: Eine empirische Untersuchung zur Person-Umwelt-Transaktion. Frankfurt, Peter Lang, 1997.

36 WAHL, H-W; MOLLENKOPF, H.; OSWALD, F. Alte Menschen in ihren räumlich-dinglichen Umwelten: Herausforderungen der ökologischen Gerontologie. In: Naegele G, Schütz R (Hrsg.). Gedenkschrift für Dr. Margret Dieck [no prelo]

37 WAHL, H-W; SAUP W. Ökologische Gerontologie: mehr als die Docility-Hypothese? Zeitschrift für Gerontologie, Darmstadt, v.27, p.347454, 1994.

38 WAHL, H-W, TESCH-RÖMER, C. Interventionsgerontologie im deutschsprachigen Raum: Eine sozial-und verhaltenswissenschaftliche Bestandsaufnahme. Zeitschrift für Gerontologie und Geriatrie, Darmstad, v. 31, p.76-88, 1988.

39 WAHL, H-W, WETZLER, R. Integrierter Gesamtbericht zur Studie "Möglichkeiten und Grenzen selbständiger Lebensführung in Privathaushalten”. Bonn, Bundesministerium für Familie, Senioren, Frauen und Jugend. Kohlhammer [no prelo]

40 WHITE, R. W. Motivation Reconsidered: the concept of competence. Psychological Review, Washington, D. C., v. 66, p.297-333, 1959.

41 WILLIS, S. L. Cognition and Everyday Competence. Annual Review of Gerontology and Geriatrics, New York, v.11, p. 80-109, 1991.

42 WILLIS, S. L. Everyday Problem Solving. In: BIRREN, J.E., SCHAIE, K.W. (Eds.). Handbook of the Psychology of Aging. $4^{\text {th }}$ ed. New York, Academic Press, 1996, p. 287-307.

43 WILLIS, S. L.; SCHAIE, K.W., HAYWARD, M. (Eds.). Societal Mechanisms for Maintaining Competence in Old Age. New York, Springer, 1997.

44 WOLINSKY, F. D.; CALLAHAN, C. M.; FITZGERALD, J. F.;JOHNSON, R. J. Changes in Functional Status and the Risks of Subsequent Nursing Home Placement and Death. Journal of Gerontology: Social Sciences, Washington, D. C., v.48, p.93-101, 1993. 
